\title{
Butorphanol Inhibits the Malignant Biological Behaviors of Ovarian Cancer Cells via Down-Regulating the Expression of TMEFFI
} This article was published in the following Dove Press journal:
OncoTargets and Therapy
Purpose: An important issue with compounds for treating ovarian cancer is the development of drug resistance and side effects. Butorphanol is a synthetic opioid. Opioids have been shown to promote or prevent tumor growth and metastasis. This research aimed to reveal the affection of Butorphanol on the malignant biological behaviors of ovarian cancer cells.

Methods: Different concentrations of Butorphanol were used to treat ovarian cancer cell lines, ES-2 and SKOV3. Biological functions of cells were performed by CCK-8 assay, colony formation assay, apoptosis analysis, transwell assays and scratch assays. The differences in the transcriptome of the Butorphanol treated and negative control (NC) cells were analyzed by RNA-Seq.

Results: Butorphanol treatment significantly inhibited the viability, colony-forming, migration and invasion of ES-2 and SKOV3 cells compared to NC. Furthermore, Butorphanol treatment obviously induced the apoptosis of ES-2 and SKOV3 cells and regulated the expression of apoptosis-related proteins. Additionally, Butorphanol treatment significantly reduced the expression of p-AKT, p-mTOR and P70S6K without affecting the expression of AKT and mTOR in ES-2 and SKOV3 cells. Forty-four genes were identified to up-regulate its expression, while 17 genes were identified to down-regulate its expression in Butorphanol-treated cells. Among them, TMEFF1 was found to be significantly downregulated in Butorphanol-treated cells. Additionally, the restoration of TMEFF1 expression complemented the inhibitory effect of Butorphanol treatment on cell proliferation and invasion.

Conclusion: In conclusion, Butorphanol is a compound with potential to treat ovarian cancer. TMEFF1 may play a key role in inhibiting the malignant proliferation and metastasis of Butorphanol treatment on ovarian cancer cells.

Keywords: Butorphanol, ovarian cancer, TMEFF1, RNA-Seq, proliferation

\section{Introduction}

Ovarian cancer is one of the most deadly gynecological malignancies in the world. ${ }^{1,2}$ About $70 \%$ of patients with ovarian cancer show peritoneal metastasis in early diagnosis. ${ }^{3}$ Although great progress has been made in the therapy of ovarian cancer in recent years, an important issue with compounds for treating tumors is the development of drug resistance and side effects. ${ }^{4,5}$ Therefore, there is an urgent need to identify improved anti-tumor drugs.

Butorphanol is a synthetic opioid that has partial agonist and partial antagonist activity to the $\mu$ type opioid receptors, as well as antagonist and partial agonist
Correspondence: Chuanfeng Zhang Email meiliyuyuan@163.com
OncoTargets and Therapy 2020:13 10973-1098I

10973 
activity at the $\mathrm{K}$ opioid receptor. ${ }^{6}$ It can be used as a nasal spray to treat migraine and parenterally as a narcotic analgesic for moderate to severe pain or as an adjunct to general anesthesia. ${ }^{6,7}$ Opioids have been documented either to promote or to prevent tumor growth and metastasis. ${ }^{8}$

TMEFF1 is a transmembrane protein consisting of a epidermal growth factor (EGF)-like region and two follistatin domains. ${ }^{9}$ One study finds the differential expression of TMEFF1 in a variety of tumors including brain cancer, pancreatic cancer and liver cancer. ${ }^{10}$ Exogenously overexpressing TMEFF1 inhibits the proliferation of brain cancer cell lines. ${ }^{10}$ In addition, differential methylation of TMEFF1 have been found in both breast and colon cancer. $^{11,12}$ These studies indicate the potential role of TMEFF1 in cancer, but require more detailed research.

In present research, we found that Butorphanol treatment significantly inhibited the malignant biological behaviors of ES-2 and SKOV3 cells. Then, TMEFF1 was found to be significantly down-regulated in Butorphanol-treated cells. Additionally, the restoration of TMEFF1 expression complemented the inhibitory effect of Butorphanol treatment on cell proliferation and invasion.

\section{Materials and Methods Cell Culture and Treatment}

Human ovarian cancer cell lines (ES-2 and SKOV3) and normal cervical cell line (HCK1T) were obtained from the Institute of Biochemistry and Cell Biology, Chinese Academy of Sciences (Shanghai, China). The cells were maintained in RPMI 1640 culture medium (Gibco, Grand Island, NY) containing 10\% FBS (Thermo Fisher Scientific, Waltham, MA) at $37{ }^{\circ} \mathrm{C}$ and $5 \% \mathrm{CO}_{2}$ in an incubator. Butorphanol tartrate injection was purchased from Hengrui Medicine Co., Ltd. (Jiangsu, China). Cells were treated with different concentrations of Butorphanol $(0,2.5,5,10,20,30,40,50,60,80,120,200 \mu \mathrm{g} / \mathrm{mL})$. The TMEFF1 cDNA sequence was cloned into the pcDNA3.1 vector. And recombinant plasmid was transfected into cells by Lipofectamine 2000 (Invitrogen, Carlsbad, CA).

\section{CCK8 Assay}

Five thousand cells were planted in each well of a 96-well plate, and incubated in different concentrations of Butorphanol. Then $10 \mu \mathrm{L}$ CCK-8 solution (Dojindo, Kumamoto, Japan) was added into each well. This was followed by the incubation of the plate for $2 \mathrm{~h}$ at $37^{\circ} \mathrm{C}$.
Finally, a microplate reader was used to measure the absorbance at $450 \mathrm{~nm}$.

\section{Colony Formation Assay}

Five hundred cells were planted in each well of a 6-well plate, and incubated with Butorphanol for 10 days. Then colonies were fixed with $10 \%$ neutral formalin for $1 \mathrm{~h}$ and dyed with crystal violet (Beyotime, Haimen, China). The cells were photographed under a microscope (Olympus, Tokyo, Japan).

\section{Transwell Assay}

For cell migration, 2000 cells were seeded into the upper chamber of transwell chambers (Corning Incorporated, Corning, NY) and $200 \mu \mathrm{L}$ of serum-free RPMI-1640 medium containing different concentrations of Butorphanol was added. The lower chamber was added with $600 \mu \mathrm{L}$ of RPMI- 1640 medium containing $10 \% \mathrm{FBS}$. After $24 \mathrm{~h}$ incubation at $37^{\circ} \mathrm{C}$, the surface of each upper chamber was wiped gently with a cotton swab. Transwell chambers were fixed with $4 \%$ paraformaldehyde at room temperature for $30 \mathrm{~min}$ and stained with crystal violet for $30 \mathrm{~min}$. The tumor cells infiltrating the filter membrane on the surface of the lower chamber were then counted under a microscope (Olympus) and the cell number is expressed as the average number of the cells in each field. For cell invasion, Matrigel (BD) was spread onto the upper wells of Transwell chambers and the other steps were consistent with cell migration experiments.

\section{Scratch Assay}

When the cell monolayer reached $90 \%$, the microplate was scratched gently with a $10 \mu \mathrm{L}$ pipette tip. After washing with PBS, medium was replaced with the serum-free culture medium containing difference concentration of Butorphanol. The width of scratch was observed under a microscope. After $24 \mathrm{~h}$ of culturing, the width of each scratch was measured under a microscope.

\section{Detection of Cell Apoptosis by Flow Cytometry}

$5 \times 10^{5}$ cells were cultured in each well of a 6-well plate, and incubated in different concentrations of Butorphanol for 48 $\mathrm{h}$. Then, cells were harvested and resuspended in binding buffer. The cells were stained with $5 \mu \mathrm{L}$ Annexin V-FITC plus $5 \mu \mathrm{L}$ propidium iodide (BD Biosciences, Franklin Lakes, NJ). The stained cells were added of $400 \mu \mathrm{L}$ PBS. The percentage of apoptosis was analyzed by using flow cytometry (Tree Star, Ashland, OR). 


\section{Western Blot}

Treated cells were dissolved with RIPA buffer at $4^{\circ} \mathrm{C}$ for 10 min. The total protein was collected by centrifugation at $12,000 \times \mathrm{g}$ for $30 \mathrm{~min}\left(4^{\circ} \mathrm{C}\right)$ and quantified using a bicinchoninic acid method (Beyotime, Shanghai, China). Proteins were separated using 10\% SDS-PAGE and transferred to a PVDF membrane (EMD Millipore, Billerica, MA). After blocking with 5\% fat-free milk for $1 \mathrm{~h}$, each primary antibody was incubated overnight at $4^{\circ} \mathrm{C}$. After washing with TBST, secondary antibody was incubated for $1 \mathrm{~h}$ at room temperature. Protein bands were developed using the ECL reagent (Thermo Fisher Scientific).

The primary antibodies used in this study were as follow: anti-Bcl2 (1:1000, ab32124), anti-Bax (1:1000, ab32503), anti-cleaved caspase3 (1:1000, ab13847), antiGAPDH (1:5000, ab8245), anti-AKT (1:5000, ab8805), anti-p-AKT (1:5000, ab38449), anti-mTOR (1:5000, ab2732), anti-p-mTOR (1:5000, ab84400), anti-P70S6K (1:5000, ab131526) were purchased from Abcam. AntiTMEFF1 (1:5000, T4829) was purchased from MERCK.

\section{Statistical Analysis}

The raw image data files obtained by high-throughput sequencing (Illumina HiSeq) were converted into raw data of sequencing sequences by CASAVA base calling analysis, and the results were stored in the FASTQ file. Bowtie2 was used to align clean reads to the reference sequence, and RSEM was used to calculate the expression levels of genes and transcripts. According to the DEseq2 method (based on the principle of negative binomial distribution), differentially expressed genes (DEG) were detected. According to the differential gene detection results, the pheatmap function in R software was used to perform hierarchical cluster analysis. The data were analyzed with the SPSS 22.0 software. Each experiment was repeated at least three times. The results were presented as mean $\pm \mathrm{SD}$. Student's $t$-test was used for comparisons between two independent groups, and one-way ANOVA was used to compare differences among more than two groups. $\mathrm{P}<0.05$ indicated that the difference was statistically significant.

\section{Results}

\section{Butorphanol Inhibits the Viability and Colony-Forming Ability of Ovarian Cancer Cells}

To reveal the affection of Butorphanol on the malignant biological behaviors of ovarian cancer cells, the viability and colony-forming ability of ovarian cancer cells were firstly examined by CCK8 and colony formation assays. As shown in Figure 1A, the results of CCK8 assay showed the absorbance at $450 \mathrm{~nm}$ of ES-2 and SKOV3 cells which were treated with different concentrations of Butorphanol for 24 h. The IC 50 of Butorphanol on ES-2 cells was $31.23 \mu \mathrm{g} / \mathrm{mL}$, while the IC 50 of Butorphanol on SKOV3 cells was 39.90 $\mu \mathrm{g} / \mathrm{mL}$. Therefore, these concentrations $(30 \mu \mathrm{g} / \mathrm{mL}$ to ES-2 cells and $40 \mu \mathrm{g} / \mathrm{mL}$ to SKOV3 cells) were used in subsequent experiments. As shown in Figure 1B, Butorphanol treatment significantly inhibited the viability of ES-2 and SKOV3 cells compared into negative control (NC) group $(P<0.05)$. Moreover, the concentration of Butorphanol was safe for normal cervical cells (Supplementary figure 1). Similarly, Butorphanol treatment obviously reduced the colony-forming ability of ES-2 and SKOV3 cells compared into $\mathrm{NC}$ group (Figure 1C, $P<0.05$ ).

\section{Butorphanol Impedes the Migration and Invasion of Ovarian Cancer Cells}

Furthermore, the affection of Butorphanol on the migration and invasion of ovarian cancer cells was measured by Transwell and scratch assays. As shown in Figure 2AC, Butorphanol treatment significantly inhibited the migration and invasion capacities of ES-2 and SKOV3 cells compared into $\mathrm{NC}$ group $(P<0.05)$.

\section{Butorphanol Induces the Apoptosis of Ovarian Cancer Cells}

Flow cytometry was used to detect the apoptosis of ovarian cancer cells. As shown in Figure 2D, the apoptotic rate was significantly increased after the Butorphanol treatment $(P<$ 0.05). In addition, the expression of Bcl 2 was decreased, while the expression of Bax and cleaved-caspase 3 was increased after the Butorphanol treatment (Figure 3A, $P<0.05)$.

\section{Butorphanol Inhibits the Activation of AKT/mTOR Signaling Pathway in Ovarian Cancer Cells}

Previous studies showed that AKT/mTOR signaling pathway is involved in various human diseases including cancers. ${ }^{13,14}$ Therefore, Western blot was utilized to measure the key proteins expression of AKT/mTOR signaling pathway. As shown in Figure 3B, Butorphanol treatment significantly reduced the expression of $\mathrm{p}-\mathrm{AKT}, \mathrm{p}-\mathrm{mTOR}$ and P70S6K $(P<0.05)$ without affecting the expression of AKT and mTOR in ES-2 and SKOV3 cells. 

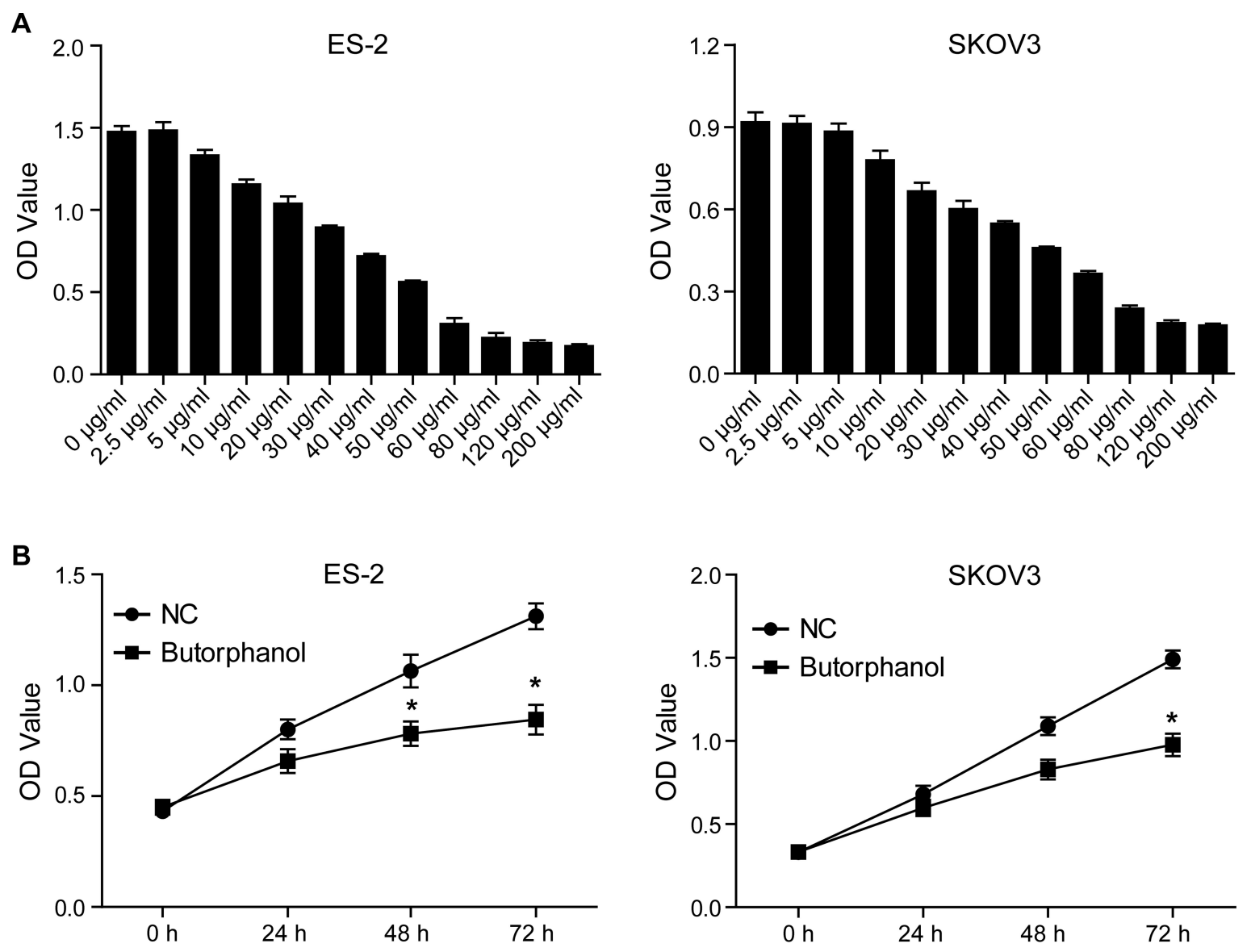

C
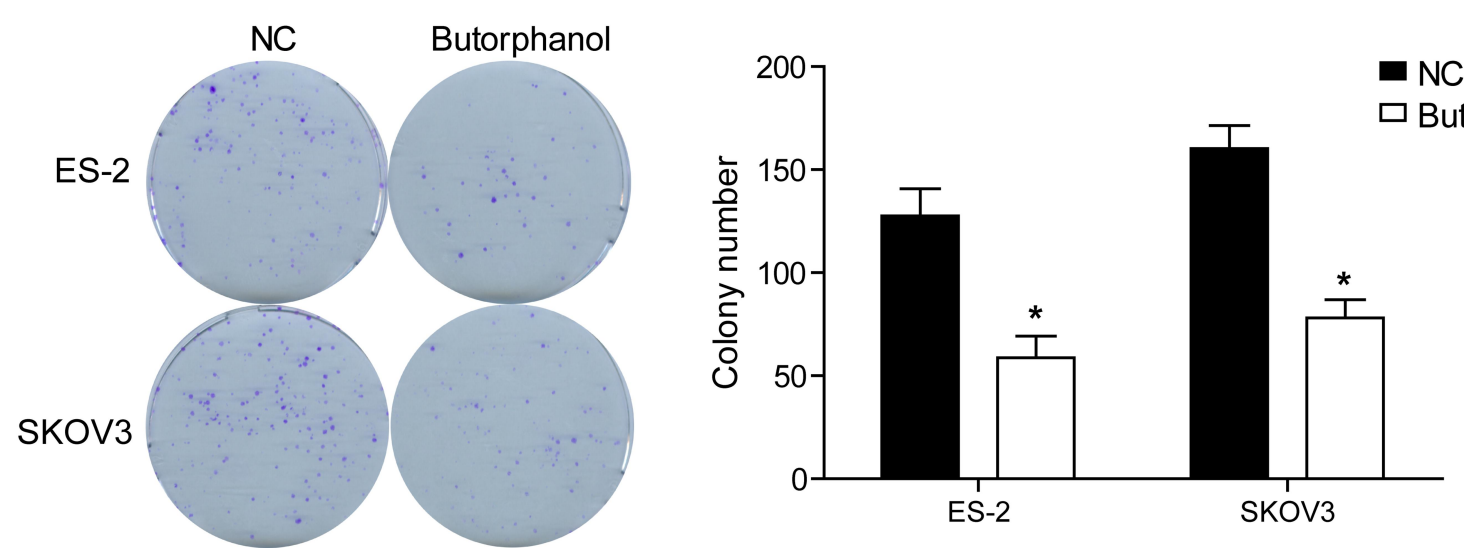

Figure I Butorphanol inhibits the viability and colony-forming ability of ovarian cancer cells.

Notes: (A) ES-2 and SKOV3 cells were treated with different concentrations of Butorphanol for 24 h, and CCK8 assay was performed to examine cell viability. (B) ES-2 cells were treated with $30 \mu \mathrm{g} / \mathrm{mL}$ of Butorphanol, while SKOV3 cells were treated with $40 \mu \mathrm{g} / \mathrm{mL}$ of Butorphanol, and CCK8 assay was performed to examine cell viability. (C) After being treated with Butorphanol, colony formation assay was performed. $* P<0.05$.

\section{Butorphanol Inhibits the Expression of TMEFFI in Ovarian Cancer Cells}

The differences in the transcriptome of the Butorphanol treated and NC cells were analyzed by RNA-Seq (Beijing Genomics Institute) (Figure 4A). qRT-PCR was used to confirm the accuracy of the analyze results (results not shown). The results of RNA-Seq performed that 44 genes were up-regulated, while 17 genes were downregulated when cells were treated with Butorphanol (Figure 4B and $\mathrm{C}$ ). Through literature search and 

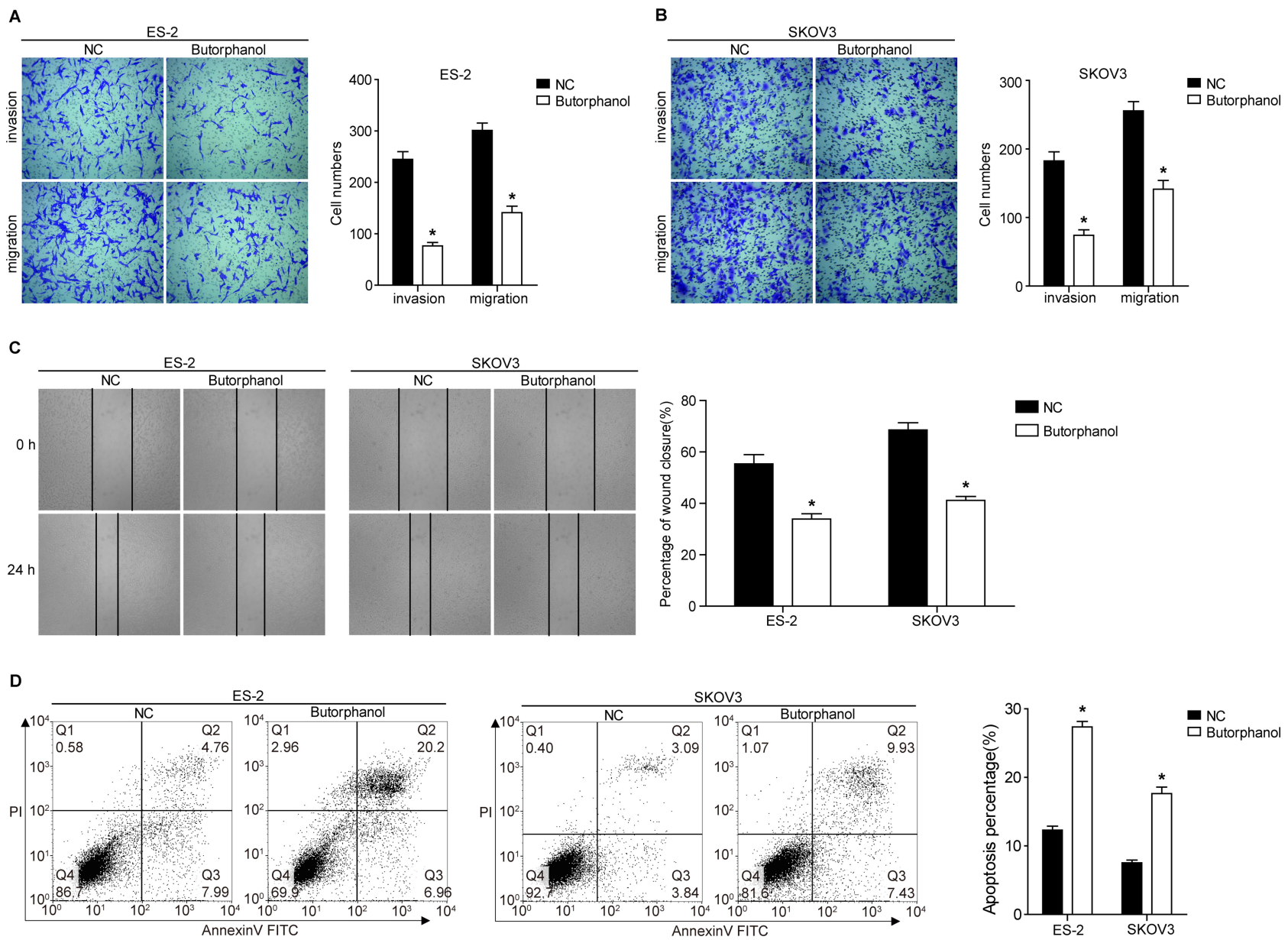

Figure 2 Butorphanol impedes the migration and invasion of ovarian cancer cells, and induces the cell apoptosis.

Notes: Cell migration and invasion in Butorphanol-treated ES-2 (A) and SKOV3 (B) cells were detected by Transwell assays. (C) Images and wound closure percentage of ES-2 and SKOV3 cells for $24 \mathrm{~h}$ after Butorphanol treatment. (D) The rate of apoptosis in ES-2 and SKOV3 cells exposed to Butorphanol for $24 \mathrm{~h}$ was detected by flow cytometry. $* P<0.05$.

experimental verification, TMEFF1 gene was selected for the subsequent research.

As shown in Figure 4D, Butorphanol treatment significantly reduced the expression of TMEFF1, while cotransfection of TMEFF1 over-expression plasmid restored the TMEFF1 expression. Additionally, the restoration of TMEFF1 expression complemented the inhibitory effect of Butorphanol treatment on cell proliferation and invasion (Figure 4E and F).

\section{Discussion}

Globally, 240,000 women are diagnosed with ovarian cancer each year, and the 5-year survival rate is less than $45 \%$, resulting in 150,000 deaths. ${ }^{15}$ Although the age-standardized ratio has stabilized or declined in most high-income countries, the age-standardized ratio has increased in many low- and middleincome countries. In addition, as life expectancy increases, the number of confirmed cases each year also increases. Therefore, there is an urgent need to identify new antitumor drugs.

Butorphanol is a competitive $\mu$-receptor antagonist that provides analgesia through an agonist effect on -receptors. ${ }^{7}$ In the United States, Butorphanol is used to treat anesthesia, anesthesia aids, labor pain and pain, as a member of the drug class of narcotic analgesics. It will provide a new possibility for cancer treatment. In this study, the affection of Butorphanol on the viability of ovarian cancer cells was firstly detected by CCK8 assays. The IC 50 of Butorphanol on ES-2 cells was $31.23 \mu \mathrm{g} / \mathrm{mL}$, while the IC 50 of Butorphanol on SKOV3 cells was 39.90 $\mu \mathrm{g} / \mathrm{mL}$. Furthermore, Butorphanol treatment significantly inhibited the proliferation, migration and invasion of ES-2 and SKOV3 cells. Additionally, Butorphanol treatment obviously induced the apoptosis of ovarian cancer cells by regulating the expression of $\mathrm{Bcl} 2, \mathrm{Bax}$ and 
A
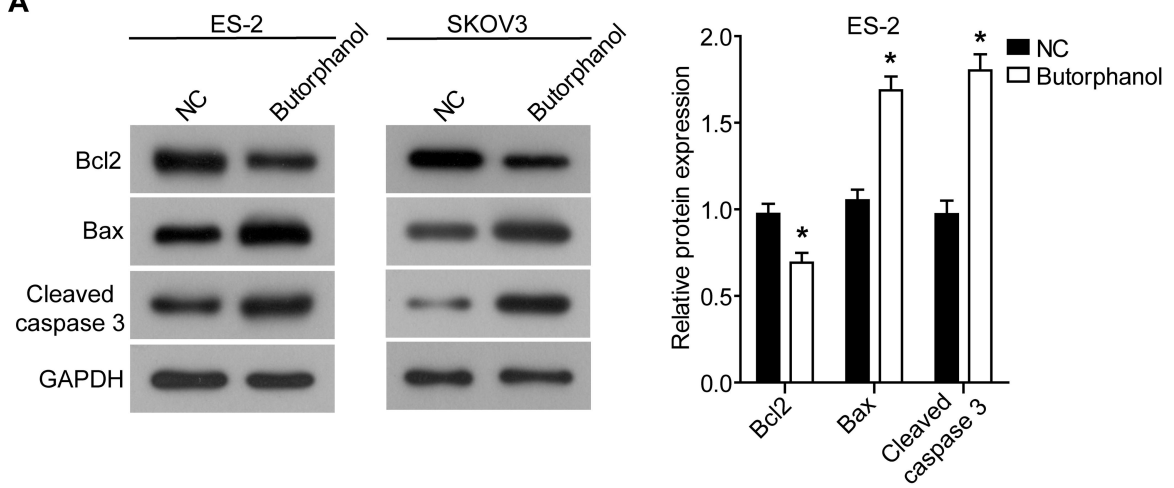

B

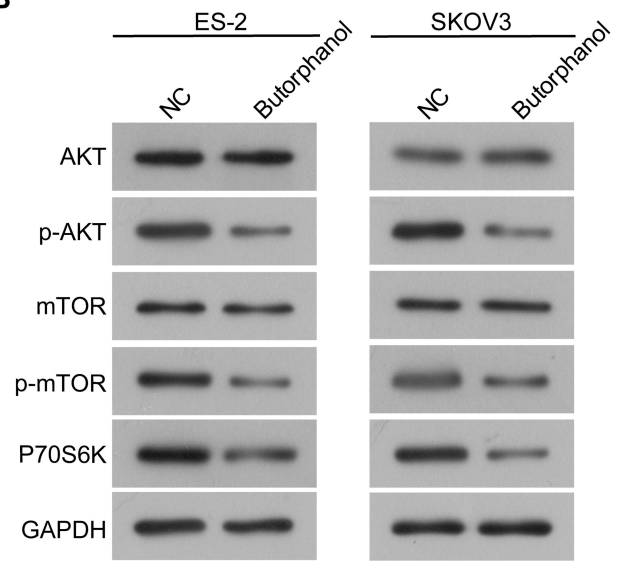



Figure 3 Butorphanol inhibits the activation of AKT/mTOR signaling pathway in ovarian cancer cells.

Notes: (A) After $48 \mathrm{~h}$ of incubation with Butorphanol, the expression of apoptosis-related proteins (Bcl-2, Bax and Cleaved caspase3) was detected by Western blot analysis. (B) The effects of Butorphanol on AKT/mTOR signaling pathway were analyzed by Western blot assay. $* P<0.05$.

cleaved-caspase 3. These data reveal that Butorphanol could inhibit ovarian cancer progression. In addition, the concentrations of Butorphanol, which significantly inhibit the proliferation of cervical cancer cells, is safe for normal cervical cells. This demonstrates the possibility of Butorphanol in the treatment of cervical cancer. Although there is still a lack of pharmacological and pharmacokinetic experiments for Butorphanol.

We also found that Butorphanol treatment inhibited the activation of $\mathrm{AKT} / \mathrm{mTOR}$ signaling pathway in ovarian cancer cells. The results of Western blot showed that Butorphanol treatment significantly reduced the expression of p-AKT, p-mTOR and P70S6K without affecting the expression of AKT and mTOR in ES-2 and SKOV3 cells. These data reveal that Butorphanol inhibits the malignant biological behaviors of ovarian cancer cells by regulating the AKT/mTOR signaling pathway, which has the potential to treat ovarian cancer. Butorphanol is a synthetic opioid. The sequencing results indicated that the cell lines (ES-2 and SKOV3) used in this study expressed opioid receptors. However, Butorphanol treatment did not cause changes in the expression of opioid receptors. The sequencing results showed that 44 genes were up-regulated its expression, while 17 genes were down-regulated its expression in Butorphanol-treated cells. The differential expression of these genes may play a key role in inhibiting the malignant proliferation and metastasis of Butorphanol treatment on ovarian cancer cells. Among them, TMEFF1 was found to be significantly down-regulated in Butorphanol-treated cells. Additionally, the restoration of TMEFF1 expression complemented the inhibitory effect of Butorphanol treatment on cell proliferation and invasion. Some recent studies find that some effects of opioids are not mediated by opioid receptors. ${ }^{8,16,17}$ For example, Morphine decreased matrix metalloprotease-9 (MMP-9) and increased tissue inhibitor of metalloproteases-1 (TIMP-1) in multiple experimental models, with consequences on tumor cell migration and invasion. $^{16,17}$ Some studies show that opioids or their 
A



B



C

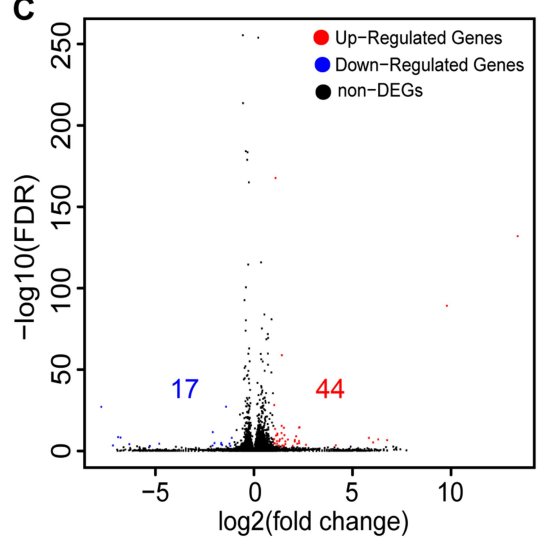

D
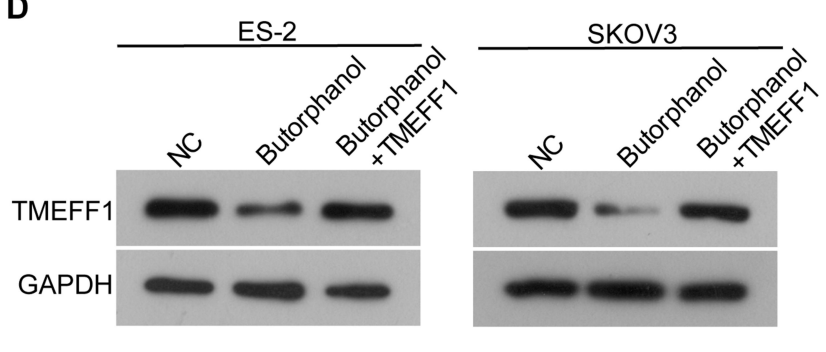

E

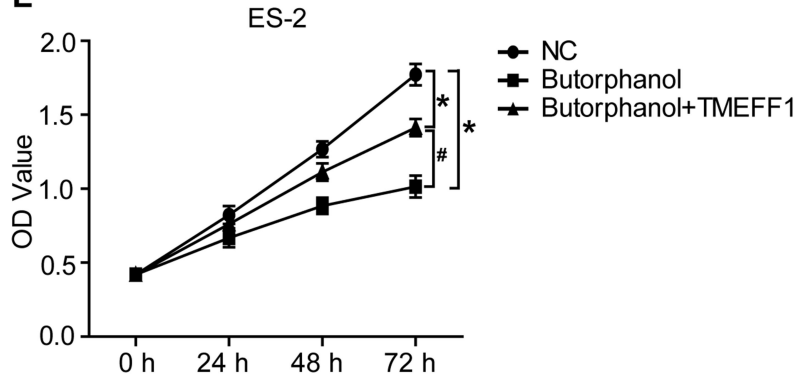

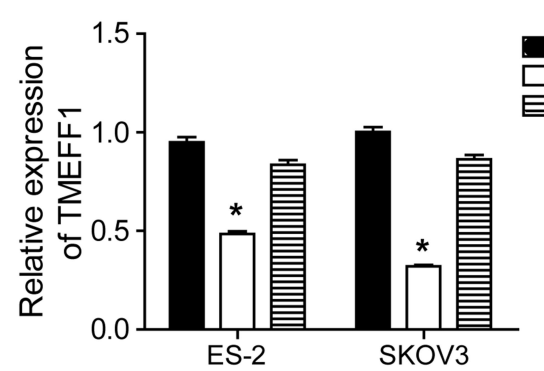

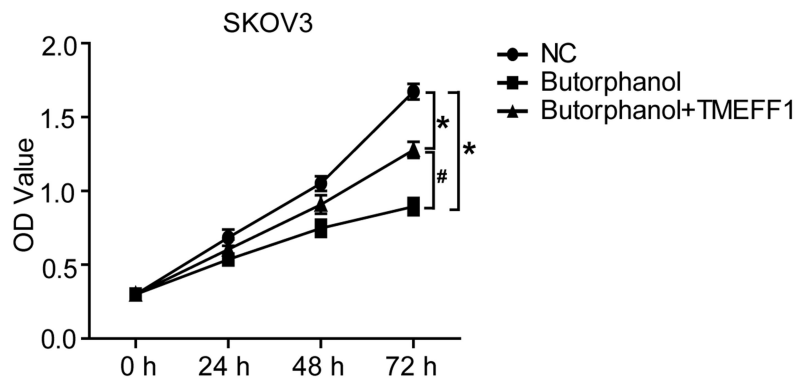

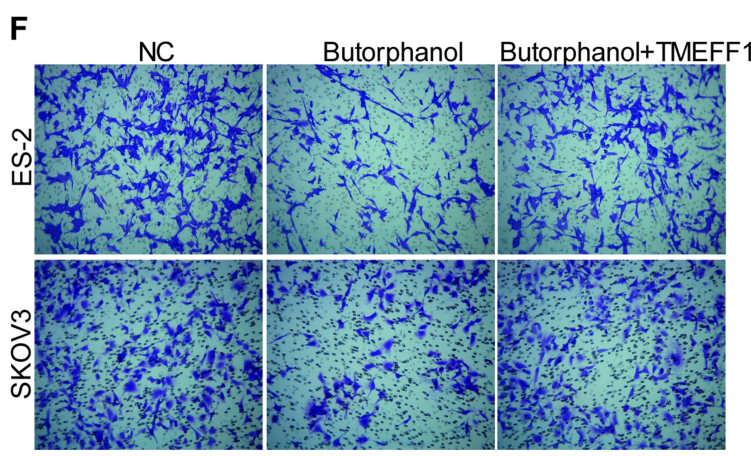



Figure 4 Butorphanol inhibits the expression of TMEFFI in ovarian cancer cells.

Notes: (A-C) The differences in the transcriptome of the Butorphanol treated and NC cells were analyzed by RNA-Seq. (D) Western blot assay was performed to examine the expression of TMEFFI. (E) CCK8 assay was used to examine cell viability of ES-2 and SKOV3 cells. (F) Cell migration and invasion were assessed by Transwell assay. ${ }^{*} P<0.05$, compared to NC group; ${ }^{\#} P<0.05$, compared to Butorphanol group.

Abbreviations: negative control, NC; non-DEGs, non-difference expression genes. 
metabolites can activate toll-like receptor 4 (TLR4), which is expressed on cancer cells and tumor-related cells and regulates metastasis signaling pathways. ${ }^{8}$ Although the sequencing results showed that Butorphanol treatment did not affect TLR4 expression. Therefore, we speculate that its effect does not seem to be related to opioid receptor and TLR4 activation.

Initial research showed that TMEFF1 is mainly expressed in the brain. ${ }^{9}$ Subsequent research found that TMEFF1 is widely expressed in a variety of tissues, and play different role. ${ }^{10}$ For example, TMEFF1 is highly expressed in normal brain tissues, and lowly expressed in brain tumor tissue. ${ }^{10}$ Exogenously overexpressing TMEFF1 inhibits the proliferation of brain cancer cell lines. ${ }^{10}$ Additionally, TMEFF1 is up-regulated in pancreatic and lung cancer cells. ${ }^{10}$ Hypermethylation of TMEFF1 is found in blood samples from patients of colon cancer. ${ }^{12}$ In TP53 mutant- and TP53 wild-type breast cancer samples, differences in methylation of $\mathrm{CpG}$ island of the TMEFF1 promoter are also found. ${ }^{11}$ Cancer-testis (CT) Ags are compelling targets for immunotherapy strategies, because they are abnormally expressed in malignant cells and not or limited expressed in somatic tissues, except germ cells. ${ }^{18}$ One study identifies TMEFF1 as a CT Ag candidate in multiple myeloma. ${ }^{19}$ These results indicate that the expression of TMEFF1 plays an important role in the occurrence and development of many types of tumors.

\section{Conclusion}

In conclusion, Butorphanol is a compound with potential to treat ovarian cancer. TMEFF1 may play a key role in inhibiting the malignant proliferation and metastasis of Butorphanol treatment on ovarian cancer cells.

\section{Ethical Approval}

Not applicable.

\section{Funding}

This study did not receive any funding.

\section{Disclosure}

All authors declare that they have no conflict of interest.

\section{References}

1. Webb PM, Jordan SJ. Epidemiology of epithelial ovarian cancer. Best Pract Res Clin Obstet Gynaecol. 2017;41:3-14. doi:10.1016/j. bpobgyn.2016.08.006

2. Siegel RL, Miller KD, Jemal A. Cancer statistics, 2018. CA Cancer J Clin. 2018;68(1):7-30. doi:10.3322/caac. 21442

3. Eisenhauer EA. Real-world evidence in the treatment of ovarian cancer. Ann Oncol. 2017;28(suppl_8):viii61viii65. doi:10.1093/annonc/mdx443

4. Turner JG, Dawson J, Sullivan DM. Nuclear export of proteins and drug resistance in cancer. Biochem Pharmacol. 2012;83 (8):1021-1032. doi:10.1016/j.bcp.2011.12.016

5. Lheureux S, Braunstein M, Oza AM. Epithelial ovarian cancer: evolution of management in the era of precision medicine. $C A$ Cancer J Clin. 2019;69(4):280-304.

6. Butorphanol. In: LiverTox: Clinical and Research Information on Drug-Induced Liver Injury. Bethesda (MD) 2012.

7. Thigpen JC, Odle BL, Harirforoosh S. Opioids: a review of pharmacokinetics and pharmacodynamics in neonates, infants, and children. Eur J Drug Metab Pharmacokinet. 2019;44(5):591-609. doi:10.1007/ s13318-019-00552-0

8. Xie N, Matigian N, Vithanage T, et al. Effect of perioperative opioids on cancer-relevant circulating parameters: mu opioid receptor and toll-like receptor 4 activation potential, and proteolytic profile. Clin Cancer Res. 2018;24(10):2319-2327. doi:10.1158/1078-0432.CCR-18-0172

9. Eib DW, Martens G. A novel transmembrane protein with epidermal growth factor and follistatin domains expressed in the hypothalamohypophysial axis of Xenopus laevis. J Neurochem.1996;67 (3): 1047-1055.

10. Gery S, Yin D, Xie D, Black KL, Koeffler HP. TMEFF1 and brain tumors. Oncogene. 2003;22(18):2723-2727. doi:10.1038/sj.onc.1206351

11. Ronneberg JA, Fleischer T, Solvang HK, et al. Methylation profiling with a panel of cancer related genes: association with estrogen receptor, TP53 mutation status and expression subtypes in sporadic breast cancer. Mol Oncol. 2011;5(1):61-76. doi:10.1016/j.molonc.2010.11.004

12. Rasmussen SL, Krarup HB, Sunesen KG, Pedersen IS, Madsen PH, Thorlacius-Ussing O. Hypermethylated DNA as a biomarker for colorectal cancer: a systematic review. Colorectal Dis. 2016;18 (6):549-561. doi:10.1111/codi.13336

13. O'Donnell JS, Massi D, Teng MWL, Mandala M. PI3K-AKT-mTOR inhibition in cancer immunotherapy, redux. Semin Cancer Biol. 2018;48:91-103. doi:10.1016/j.semcancer.2017.04.015

14. Yu JS, Cui W. Proliferation, survival and metabolism: the role of PI3K/ $\mathrm{AKT} / \mathrm{mTOR}$ signalling in pluripotency and cell fate determination. Development. 2016;143(17):3050-3060. doi:10.1242/dev.137075

15. La Vecchia C. Ovarian cancer: epidemiology and risk factors. Eur J Cancer Prev. 2017;26(1):55-62. doi:10.1097/CEJ.0000000000000217

16. Xie N, Khabbazi S, Nassar ZD, et al. Morphine alters the circulating proteolytic profile in mice: functional consequences on cellular migration and invasion. FASEB J. 2017;31(12):5208-5216.

17. Khabbazi S, Goumon Y, Parat MO. Morphine modulates interleukin-4- or breast cancer cell-induced pro-metastatic activation of macrophages. Sci Rep. 2015;5:11389. doi:10.1038/srep11389

18. Scanlan MJ, Simpson AJ, Old LJ. The cancer/testis genes: review, standardization, and commentary. Cancer Immun. 2004;4:1.

19. Condomines M, Hose D, Reme T, et al. Gene expression profiling and real-time PCR analyses identify novel potential cancer-testis antigens in multiple myeloma. J Immunol. 2009;183(2):832-840. doi:10.4049/jimmunol.0803298 


\section{Publish your work in this journal}

OncoTargets and Therapy is an international, peer-reviewed, open access journal focusing on the pathological basis of all cancers, potential targets for therapy and treatment protocols employed to improve the management of cancer patients. The journal also focuses on the impact of management programs and new therapeutic

Submit your manuscript here: https://www.dovepress.com/oncotargets-and-therapy-journal agents and protocols on patient perspectives such as quality of life, adherence and satisfaction. The manuscript management system is completely online and includes a very quick and fair peer-review system, which is all easy to use. Visit http://www.dovepress.com/ testimonials.php to read real quotes from published authors. 